\title{
Active Control of the THz Wave Polarization State by an Electronically Controlled Graphene Composite Metasurface
}

\author{
Guocui Wang ${ }^{1,2}$, Bin $\mathrm{Hu}^{1 *}$, Muhammad Ismail Khan ${ }^{1}$ and Yan Zhang ${ }^{2}$ \\ ${ }^{1}$ School of Optics and Photonics, Beijing Engineering Research Center for Mixed Reality and Advanced Display, Beijing Institute of \\ Technology, Beijing, China, ${ }^{2}$ Department of Physics, Beijing Key Lab for Metamaterials and Devices, Key Laboratory for Terahertz \\ Optoelectronics, Ministry of Education, Beijing Advanced Innovation Center for Imaging Theory and Technology, Capital Normal \\ University, Beijing, China
}

OPEN ACCESS

Edited by: Meng Chen,

Tsinghua University, China

Reviewed by: Lei Hou,

Xi'an University of Technology, China

Xinlong $\mathrm{Xu}$,

Northwest University, China

*Correspondence:

$\mathrm{Bin} \mathrm{Hu}$

hubin@bit.edu.cn

Specialty section:

This article was submitted to

Optics and Photonics,

a section of the journal

Frontiers in Physics

Received: 31 July 2021

Accepted: 24 August 2021

Published: 06 September 2021

Citation:

Wang G, Hu B, Khan Ml and Zhang Y (2021) Active Control of the $\mathrm{THz}$ Wave Polarization State by an Electronically Controlled Graphene

Composite Metasurface.

Front. Phys. 9:751026.

doi: 10.3389/fphy.2021.751026
Active control of terahertz ( $\mathrm{THz}$ ) wave polarization state is of great significance for sensitive detection, imaging and communication. Here, a tunable $\mathrm{THz}$ quarter wave plate is designed by electronically controlling a composite metasurface consisting of the gold cross antennas and a monolayer graphene. The graphene composite metasurface acts as a quarter-wave plate when the chemical potential of graphene is $0 \mathrm{eV}$, by which the polarization state of the incident $\mathrm{THz}$ wave is converted from linear polarization to circular polarization. After the chemical potential of graphene is increased gradually, and to $0.5 \mathrm{eV}$, the transmitted polarization state of the $\mathrm{THz}$ wave is changed from right circular polarization to right elliptical polarization, and to linear polarization. Furthermore, the polarization state of the $\mathrm{THz}$ wave is able to be changed from left circular polarization to left elliptical polarization, and to linear polarization if the device is clockwise rotated by $90^{\circ}$. Therefore, the polarization state of $\mathrm{THz}$ wave could be actively controlled by the proposed tunable THz quarter wave plate. Our work will offer a new avenue for tunable $\mathrm{THz}$ polarization modulation devices.

Keywords: THz, polarization, tunable, graphene, metasurface

\section{INTRODUCTION}

Recently, tunable metasurfaces have attracted enormous research interests as a means to control the polarization state of $\mathrm{THz}$ wave. In the conventional optical devices, polarization control of electromagnetic wave is mainly achieved using birefringent materials. However, these materials are not suitable in the $\mathrm{THz}$ band because of the inherent disadvantages including bulky size, and narrow band of operating frequency. The most important issue is that the $\mathrm{THz}$ wave interacts weakly with these materials in nature.

Metasurface is a sub-wavelength artificial structure with extraordinary properties that nature materials do not have [1]. The electromagnetic wave properties including amplitude, phase, frequency and polarization can be controlled completely based on the structure of a metasurface, because any permittivity and permeability can be achieved after the modulation of metasurface devices. Therefore, more and more people have been committed to design metasurface structures, and proposed various functional devices including the anomalous reflection or refraction devices $[2,3]$, the holographic devices [4-6], the metasurface lenses [7-9], and the polarization-control devices [10-17], which mainly include the 


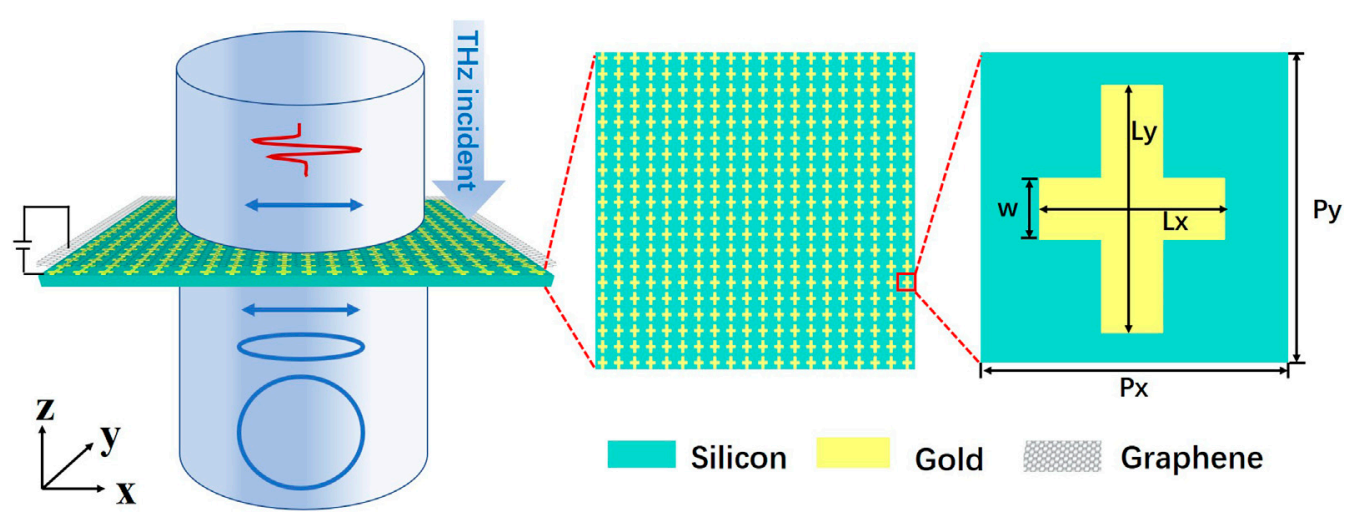

FIGURE 1 | (A) Diagram of the working principle of the tunable TQWP; (B) Schematic of the metasurface consisting of the gold anisotropy cross arrays; (C) Schematic of one unit cell among the gold gross arrays.

half-wave plate $[10,11]$, the quarter-wave plate [12-16], and the retarders [17]. These devices have the advantages of ultrathin thickness, high degree of design freedom and compactness, which will promote the miniaturization of the system. For example, Wang et al. designed an ultrathin $\mathrm{THz}$ quarter-wave plate using Babinetinverted metasurface [14]. However, all the devices mentioned above are static, which hinders their convenience in the realistic application. Activating the functionalities of these devices will no doubt expand the application and improve the functional value, which is necessary to integrate tunable materials including the semiconductor $[18,19]$, the phase change material $[20,21]$, and graphene $[22,23]$, and externally control the functionalities by light [24], temperature [25], and an applied gate voltage [26]. More recently, a few $\mathrm{THz}$ polarization devices have been proposed [27-29]. Zhao et al. proposed a tunable transmission $\mathrm{THz}$ waveplate based on the electrically driving metasurface [28]. Luo et al. designed a dynamically reversible and strong circular dichroism based on Babinet-invertible chiral metasurface [29]. However, polarization devices whose function is tunable in the $\mathrm{THz}$ band is still vary lacking.

In this paper, we design a tunable $\mathrm{THz}$ quarte-wave plate (TQWP) by integrating a monolayer graphene with a metasurface consisting of the anisotropic gold cross arrays for actively and completely controlling the $\mathrm{THz}$ polarization state. The resonance characteristics of the cross will be modulated when the Fermi energy of the graphene is changed by an external voltage [30, 31]. A polarization state conversion from right circular polarization undergoes right elliptical polarization, and to linear polarization of $45^{\circ}$ is achieved by changing the chemical potential of graphene from 0 to $0.5 \mathrm{eV}$, in which the device is irradiated normally by a linear polarized $\mathrm{THz}$ plane wave with a polarization angle of $45^{\circ}$ to the $\mathrm{x}$-axis. We further achieved the $\mathrm{THz}$ polarization conversion from left circular polarization state undergoes left elliptical polarization state, and to linear polarized of $135^{\circ}$ only making the device clockwise rotate by $90^{\circ}$.

\section{DESIGN OF STRUCTURE}

The tunable TQWP is designed as shown in Figure 1A. The device is irradiated normally by a linear polarized $\mathrm{THz}$ plane wave. After modulated by this device, the $\mathrm{THz}$ polarization state is transformed to a circular state at first. Then, the polarization state of $\mathrm{THz}$ is changed from circular polarization goes through elliptical polarization and eventually returns to linear polarization with a changed gate voltage that increases the Fermi energy of the graphene. The tunable TQWP is consisting of three parts, including the monolayer graphene, the metasurface, and the silicon. The metasurface is composed of the anisotropic gold cross arrays, as shown in Figure 1B, and the unit cell of the metasurface is depicted in Figure 1C. The period of the unit cell is $100 \mu \mathrm{m}$. The arm lengths of the cross are $L x=66 \mu \mathrm{m}$ and $L y=86 \mu \mathrm{m}$, respectively. The arm width is $w=9 \mu \mathrm{m}$. The Finite-Difference Time-Domain (FDTD) method of simulation software (FDTD Solutions, Lumerical Inc.) is used as the simulation tool to calculate the electromagnetic field of the $\mathrm{THz}$ wave. In the simulations, the graphene layer is modeled as a two-dimensional (2D) graphene sheet, whose Fermi energy is tuned by changing the graphene chemical potential. The silicon with a refractive index of 3.4 is used as a substrate of the device.

\section{RESULTS AND DISCUSSION}

Numerical simulations are then carried out to confirm the functions of the designed graphene composite metasurface. Graphene is a great popular functional material in tunable metasurface because the electronic characteristics are controlled by external stimuli. In the $\mathrm{THz}$ region, graphene is well described by the Drude-like surface conductivity as [32].

$\sigma_{\text {graphene }}\left(\omega, \mu_{c}, \Gamma, T\right)=-j \frac{e^{2} k_{B} T}{\pi \hbar^{2}(\omega-j 2 \Gamma)}\left(\frac{\mu_{c}}{k_{B} T}+2 \operatorname{In}\left(e^{-\mu_{c} / k_{B} T}+1\right)\right)$

where $e, k_{B}$ and $\hbar$ are universal constants representing the electron charge, Boltzmann's and Plank's constant, $\omega$ is the working radian frequency, $\mathrm{T}$ is the room temperature, $\Gamma$ ( $\Gamma=\hbar / 2 \tau$, where $\tau$ is the electron-photon relaxation time) is the scattering rate, and $\mu_{c}$ is the chemical potential of the 

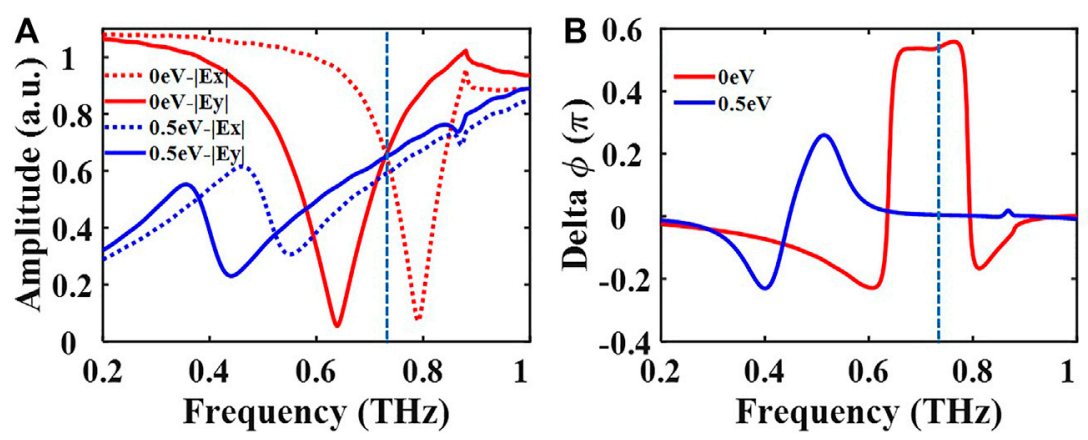

FIGURE 2 | (A) Variations of the transmission amplitude components $\left|E_{y}\right|$ (solid line) and $\left|E_{x}\right|$ (dashed line) when the chemical potential of graphene was increased from 0 to $0.5 \mathrm{eV}$, respectively; (B) Phase differences between $\varnothing_{y}$ and $\varnothing_{x}$ when the chemical potential of graphene was increased from 0 to 0.5 eV, respectively.
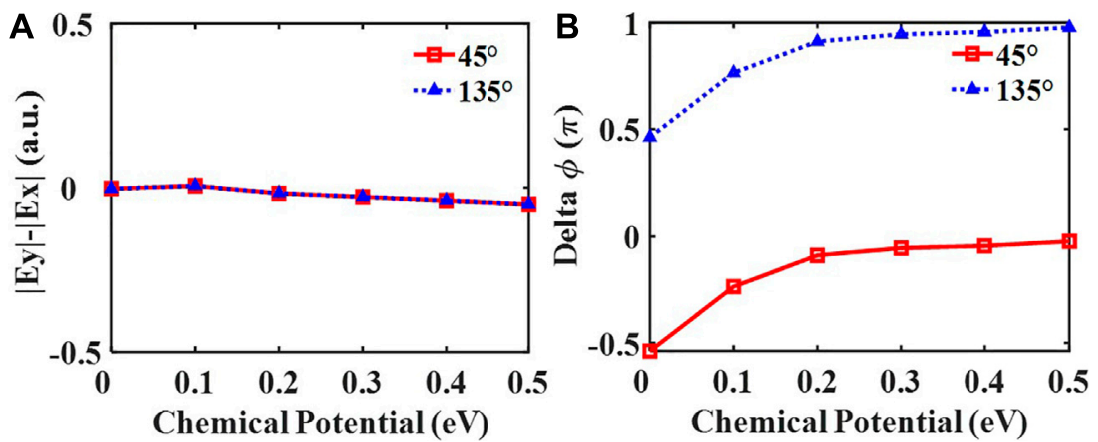

FIGURE 3 | (A) The amplitude difference $\left(\left|E_{y}\right|-\left|E_{x}\right|\right)$ between the transmission components $E_{y}$ and $E_{x}$ for THz at frequency of 0.73 THz with incident polarize angle of $45^{\circ}$ (square dotted line) and $135^{\circ}$ (triangle dotted line) when the chemical potential of graphene was increased form $0-0.5$ eV, respectively. (B) The phase difference $\left(\varnothing_{y}-\varnothing_{x}\right)$ between the transmission components $E_{y}$ and $E_{x}$ for $\mathrm{THz}$ at frequency of $0.73 \mathrm{THz}$ with incident polarize angle of $45^{\circ}$ (square dotted line) and $135^{\circ}$ (triangle dotted line) when the chemical potential of graphene was increased form 0-0.5 eV, respectively.

graphene. Therefore, the conductivity of graphene can be controlled by changing the chemical potential in the simulation, and the resonance characters affected by conductivity of the composite metasurface are controlled. In our simulation, the transmission characters were obtained for different graphene chemical potential changed from 0.1 to $0.5 \mathrm{eV}$ by a step of $0.1 \mathrm{eV}$.

Red lines in Figures 2A,B show the simulated transmission amplitude components $\left|E_{y}\right|$ (solid line) and $\left|E_{x}\right|$ (dashed line) and the difference between phase components $\varnothing_{y}$ and $\varnothing_{x}$ along the two arms of the cross in the metasurface before the chemical potential of the graphene is changed $\left(E_{F}=0 \mathrm{eV}\right)$, respectively. It is found that there are two resonant dips in the transmission amplitude components alone the two arms of the cross, respectively. If we set our sights at frequency of the $0.73 \mathrm{THz}$, we can observe that both the transmission amplitudes of the two arms are 0.67 , and the phase delay is $90^{\circ}$, which demonstrates that a function of a quarter wave plate is obtained. When the chemical potential of the graphene is increased to $0.5 \mathrm{eV}$, the transmission amplitude components and the difference between phase components are illustrated by the blue lines in Figures 2A,B, respectively. Both resonant dips of the transmission amplitude corresponding to the two arms are red-shifted. The transmission amplitudes of the two arms are about 0.60 except for some negligible differences between the two components at the frequency of $0.73 \mathrm{THz}$, but the phase delay is reduced to $0^{\circ}$, which means that the polarization of the transmission $\mathrm{THz}$ wave is back to the linear polarization state.

In order to show the variation process of the transmitted $\mathrm{THz}$ polarization state along with the change of the graphene chemical potential, we calculated the difference between the transmission amplitude components, $\left|E_{y}\right|-\left|E_{x}\right|$, and the difference between the phase components, $\varnothing_{y}-\varnothing_{x}$, of the cross, respectively, at the frequency of $0.73 \mathrm{THz}$, when the graphene chemical potential is changed from 0 to $0.5 \mathrm{eV}$ by a step of $0.1 \mathrm{eV}$. As shown by the red lines in Figures $\mathbf{3 A}, \mathbf{B}$, the difference between the two transmission amplitude components is almost zero, and the phase difference between the two transmission components is gradually changed from $-\pi / 2$ to $0 \pi$ at the incident polarization with the polarize angle of $45^{\circ}$, which indicates that the polarization state of $\mathrm{THz}$ wave changes from the right circular polarization to the right elliptical polarization, and back to the 


\begin{tabular}{|c|c|c|c|c|c|c|c|}
\hline $\begin{array}{c}\text { Incident } \\
\text { Polarization } \\
\left(^{\circ}\right)\end{array}$ & $\begin{array}{c}\text { Chemical } \\
\text { Potential } \\
\text { (eV) }\end{array}$ & Number & so & si & s2 & $\$ 3$ & Poincare Sphere \\
\hline \multirow{6}{*}{$45^{\circ}$} & 0 & 1 & 0.86 & 0.00 & -0.10 & -0.86 & \\
\hline & 0.1 & 2 & 0.69 & 0.01 & 0.51 & -0.46 & \\
\hline & 0.2 & 3 & 0.76 & 0.02 & 0.73 & -0.21 & \\
\hline & 0.3 & 4 & 0.71 & 0.03 & 0.70 & -0.12 & \\
\hline & 0.4 & 5 & 0.60 & 0.04 & 0.59 & -0.08 & \\
\hline & 0.5 & 6 & 0.52 & 0.05 & 0.51 & -0.03 & \\
\hline \multirow{6}{*}{$135^{\circ}$} & 0 & 7 & 0.86 & 0.00 & 0.10 & 0.86 & \\
\hline & 0.1 & 8 & 0.70 & 0.01 & -0.51 & 0.46 & \\
\hline & 0.2 & 9 & 0.76 & 0.02 & -0.73 & 0.21 & \\
\hline & 0.3 & 10 & 0.71 & 0.03 & -0.70 & 0.12 & \\
\hline & 0.4 & 11 & 0.60 & 0.04 & -0.60 & 0.08 & \\
\hline & 0.5 & 12 & 0.52 & 0.05 & -0.51 & 0.03 & \\
\hline
\end{tabular}

FIGURE 4 | (Left column) The Stokes Parameters of the devices for the different graphene chemical potential with the $\mathrm{THz}$ incidence polarize angle $45^{\circ}$ and $135^{\circ}$, respectively. (Right column) The Poincare Sphere is used to presents the polarization state of the device for different chemical potential of graphene.

linear polarization with the polarize angle of $45^{\circ}$. As shown by the blue lines in the Figures $\mathbf{3 A}, \mathbf{B}$, the difference between two transmission amplitude components is almost zero, and the phase difference between the two transmission components is gradually changed from $\pi / 2$ to $\pi$ only by rotating the sample of $90^{\circ}$ clockwise, taking the $\mathrm{z}$-axis as the center axis. In this situation, the polarization of the $\mathrm{THz}$ wave changes from the left circular polarization to the left elliptical polarization, and to the linear polarization with the polarize angle of $135^{\circ}$.

The polarization information of the transmitted wave can be represented by the Stokes parameters $\left[S_{0}, S_{1}, S_{2}, S_{3}\right]$, in which $S_{0}=\left|E_{x}\right|^{2}+\left|E_{y}\right|^{2}, S_{1}=\left|E_{x}\right|^{2}-\left|E_{y}\right|^{2}, S_{2}=\left|E_{x}\right| *\left|E_{y}\right| * \sin (\Delta \varnothing)$, and $S_{2}=\left|E_{x}\right| *\left|E_{y}\right| * \cos (\Delta \varnothing)$ [28]. From the simulated amplitude and phase of the both $E_{x}$ and $E_{y}$ components, we calculated the Stokes parameters of the designed metasurface for different graphene chemical potentials, as shown in Figure 4 (Left column). The
Poincare sphere is a way that can intuitively represents the polarization state of light, in which the north point represents the left circular polarization state, the south point represents the right circular polarization state, the equator line represents linear polarization state, the northern hemisphere represents the left elliptical polarization state, and the southern hemisphere represents the right elliptical polarization state, respectively. Therefore, the Stokes parameters calculated above is then represented by the Poincare sphere in Figure 4 (Right column). It is easily to find that the polarization state of $\mathrm{THz}$ incident with polarize angle of $45^{\circ}$ is modulated from the right circular polarization to the right elliptical polarization, and to the linear polarization when the chemical potential is changed from 0 to $0.5 \mathrm{eV}$. Furthermore, the polarization state of $\mathrm{THz}$ incident with polarize angle of $135^{\circ}$ is modulated from the left circular polarization to the left elliptical polarization, and to the linear polarization. Therefore, the polarization of the $\mathrm{THz}$ wave can be controlled flexibly and completely by the tunable TQWP designed in this letter.

To further understand the physics underlying inside of the proposed tunable metasurface, in Figures 5A,B, we stimulated the energy distribution on the gold cross for different graphene chemical potentials of 0 and $0.5 \mathrm{eV}$, respectively. As shown in Figure $\mathbf{5 A}$, the $\mathrm{THz}$ energy is uniformly distributing on the two arms of the whole cross, respectively, the energy distribution on the arm along with the $x$-axis is stronger than that on the arm along with the y-axis, with energy intensity of $7 \times 10^{-1}$ when chemical potential of graphene is $0 \mathrm{eV}$. However, the $\mathrm{THz}$ energy is mainly distributing the end of arms of the cross, when the chemical potential of graphene is increased to $0.5 \mathrm{eV}$, as shown in Figure 5B. Furthermore, the energy intensity is reduced to $5.7 \times 10^{-2}$ which is much smaller than that energy intensity of $7 \times 10^{-1}$. The above results indicating that interaction between the $\mathrm{THz}$ wave and the gold antenna will be gradually suppressed when the chemical potential of graphene is increasing, which results in reduction of the difference between phase components. Therefore, the polarization state of $\mathrm{THz}$ is actively controlled by the designed metasurface alone with the variation of the graphene chemical potential.
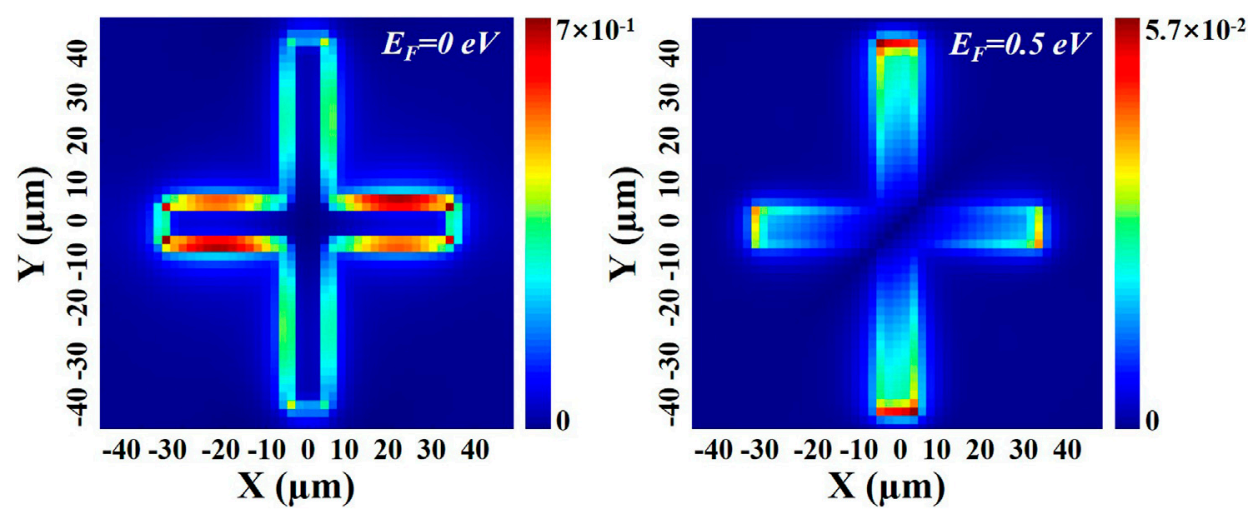

FIGURE 5 | (A,B) The THz energy distribution on the gold cross for graphene chemical potentials of 0 and 0.5 eV, respectively. 


\section{CONCLUSION}

In summary, we have demonstrated a tunable TQWP which is realized by integrating the monolayer graphene with a metallic metasurface. This composite device acts as a TQWP at the frequency of $0.73 \mathrm{THz}$ before the chemical potential of graphene is changed, by which the polarization state of $\mathrm{THz}$ with incident polarize angle of $45^{\circ}$ to $x$-axis is converted from linear polarization to circular polarization. When the chemical potential of the graphene is increased from 0 to $0.5 \mathrm{eV}$, the polarization state of the $\mathrm{THz}$ wave is converted from the right circular polarization to the right elliptical polarization, and to the linear polarization state of $45^{\circ}$. And we further achieved the polarization conversion from the left circular polarization state to the left elliptical polarization, and to the linear polarization state of $135^{\circ}$ by rotating the devise clockwise, taking the $\mathrm{z}$-axis as the center axis. A total polarization state conversion is achieved by our composite metasurface. We hope our work can further enriches the $\mathrm{THz}$ polarization devices, and provide a new route for designing the tunable polarization devices.

\section{REFERENCES}

1. Khorasaninejad M, and Capasso F. Metalenses: Versatile Multifunctional Photonic Components. Science (2017) 358(6367):eaam8100. doi:10.1126/ science.aam 8100

2. Li Z, Palacios E, Butun S, and Aydin K. Visible-Frequency Metasurfaces for Broadband Anomalous Reflection and High-Efficiency Spectrum Splitting. Nano Lett (2015) 15(3):1615-21. doi:10.1021/nl5041572

3. Li Y, Zhang J, Zhang Y, Chen $\mathrm{H}$, and Fan Y. Wideband, Co-polarization Anomalous Reflection Metasurface Based on Low-Q Resonators. Appl Phys A (2016) 122(9):851. doi:10.1007/s00339-016-0374-3

4. Pfeiffer C, and Grbic A. Controlling Vector Bessel Beams with Metasurfaces[J]. Phys Rev Appl (2014) 2(4):044012. doi:10.1103/physrevapplied.2.044012

5. He J, Wang X, Hu D, Ye J, Feng S, Kan Q, et al. Generation and Evolution of the Terahertz Vortex Beam. Opt Express (2013) 21(17):20230-9. doi:10.1364/ oe. 21.020230

6. Guo J-Y, Wang X-K, He J-W, Zhao H, Feng S-F, Han P, et al. Generation of Radial Polarized Lorentz Beam with Single Layer Metasurface. Adv Opt Mater (2018) 6(1):1700925. doi:10.1002/adom.201700925

7. Jiang X-Y, Ye J-S, He J-W, Wang X-K, Hu D, Feng S-F, et al. An Ultrathin Terahertz Lens with Axial Long Focal Depth Based on Metasurfaces. Opt Express (2013) 21(24):30030-8. doi:10.1364/oe.21.030030

8. Wang $\mathrm{B}, \mathrm{Wu} \mathrm{X}$, and Zhang $\mathrm{Y}$. Multiple-Wavelength Focusing and Demultiplexing Plasmonic Lens Based on Asymmetric Nanoslit Arrays. Plasmonics (2013) 8(4):1535-41. doi:10.1007/s11468-013-9569-Z

9. Ho JS, Qiu B, Tanabe Y, Yeh AJ, Fan S, and Poon ASY. Planar Immersion Lens with Metasurfaces. Phys Rev B (2015) 91(12):125145. doi:10.1103/ physrevb.91.125145

10. Roberts A, and Lin L. Plasmonic Quarter-Wave Plate. Opt Lett (2012) 37(11): 1820-2. doi:10.1364/ol.37.001820

11. Pors A, Nielsen MG, and Bozhevolnyi SI. Broadband Plasmonic Half-Wave Plates in Reflection. Opt Lett (2013) 38(4):513-5. doi:10.1364/ol.38.000513

12. Zhao $\mathrm{Y}$, and Alù A. Tailoring the Dispersion of Plasmonic Nanorods to Realize Broadband Optical Meta-Waveplates. Nano Lett (2013) 13(3):1086-91. doi:10.1021/nl304392b

13. Jiang ZH, Lin L, Ma D, Yun S, Werner DH, Liu Z, et al. Broadband and Wide Field-Of-View Plasmonic Metasurface-Enabled Waveplates. Sci Rep (2014) 4: 7511. doi:10.1038/srep07511

14. Wang D, Gu Y, Gong Y, Qiu C-W, and Hong M. An Ultrathin Terahertz Quarter-Wave Plate Using Planar Babinet-Inverted Metasurface. Opt Express (2015) 23(9):11114-22. doi:10.1364/oe.23.011114

\section{DATA AVAILABILITY STATEMENT}

The original contributions presented in the study are included in the article/Supplementary Material, further inquiries can be directed to the corresponding author.

\section{AUTHOR CONTRIBUTIONS}

$\mathrm{GW}, \mathrm{BH}$ and $\mathrm{YZ}$ concived the ideas, $\mathrm{BH}$ and $\mathrm{YZ}$ improved the ideas, GW conducted the design, mumerical simulations. GW prepared the manuscript. $\mathrm{BH}$ and $\mathrm{MK}$ supervised the project. All the authors discussed and analyzed the results.

\section{FUNDING}

National Natural Science Foundation of China (62050410347, 61875010).

15. Li Z, Liu W, Cheng H, Chen S, and Tian J. Realizing Broadband and Invertible Linear-To-Circular Polarization Converter with Ultrathin Single-Layer Metasurface. Sci Rep (2015) 5:18106. doi:10.1038/srep18106

16. Jiang Y, Wang L, Wang J, Akwuruoha CN, and Cao W. Ultra-Wideband HighEfficiency Reflective Linear-To-Circular Polarization Converter Based on Metasurface at Terahertz Frequencies. Opt Express (2017) 25(22):27616-23. doi:10.1364/oe.25.027616

17. Pors A, Nielsen MG, Valle GD, Willatzen M, Albrektsen O, and Bozhevolnyi SI. Plasmonic Metamaterial Wave Retarders in Reflection by Orthogonally Oriented Detuned Electrical Dipoles. Opt Lett (2011) 36(9):1626-8. doi:10.1364/ol.36.001626

18. Sarma R, Campione S, Goldflam M, Shank J, Noh J, Le LT, et al. A Metasurface Optical Modulator Using Voltage-Controlled Population of Quantum Well States. Appl Phys Lett (2018) 113(20):201101. doi:10.1063/1.5055013

19. Zhang Y, Zhao Y, Liang S, Zhang B, Wang L, Zhou T, et al. Large Phase Modulation of THz Wave via an Enhanced Resonant Active HEMT Metasurface. J Nanophotonics (2018) 8(1):153-70. doi:10.1515/nanoph-2018-0116

20. He J, Xie Z, Sun W, Wang X, Ji Y, Wang S, et al. Terahertz Tunable Metasurface Lens Based on Vanadium Dioxide Phase Transition. Plasmonics (2016) 11(5):1285-90. doi:10.1007/s11468-015-0173-2

21. Liu X, Wang Q, Zhang X, Li H, Xu Q, Xu Y, et al. Thermally Dependent Dynamic Meta-Holography Using a Vanadium Dioxide Integrated Metasurface. Adv Opt Mater (2019) 7(12):1900175. doi:10.1002/adom.201900175

22. Fang Z, Wang Y, Schlather AE, Liu Z, Ajayan PM, de Abajo FJ, et al. Active Tunable Absorption Enhancement with Graphene Nanodisk Arrays. Nano Lett (2014) 14(1):299-304. doi:10.1021/nl404042h

23. Dai S, Ma Q, Liu MK, Andersen T, Fei Z, Goldflam MD, et al. Graphene on Hexagonal boron Nitride as a Tunable Hyperbolic Metamaterial. Nat Nanotech (2015) 10:682-6. doi:10.1038/nnano.2015.131

24. Guo J, Wang T, Zhao H, Wang X, Feng S, Han P, et al. Reconfigurable Terahertz Metasurface Pure Phase Holograms. Adv Opt Mater (2019) 7(10): 1801696. doi:10.1002/adom.201801696

25. Wang T, He J, Guo J, Wang X, Feng S, Kuhl F, et al. Thermally Switchable Terahertz Wavefront Metasurface Modulators Based on the Insulator-ToMetal Transition of Vanadium Dioxide. Opt Express (2019) 27(15):20347. doi:10.1364/oe.27.020347

26. Huang Y-W, Lee HWH, Sokhoyan R, Pala RA, Thyagarajan K, Han S, et al. Gate-Tunable Conducting Oxide Metasurfaces. Nano Lett (2016) 16(9): 5319-25. doi:10.1021/acs.nanolett.6b00555

27. Wang D, Zhang L, Gu Y, Mehmood MQ, Gong Y, Srivastava A, et al. Switchable Ultrathin Quarter-Wave Plate in Terahertz Using Active PhaseChange Metasurface. Sci Rep (2015) 5:15020. doi:10.1038/srep15020 
28. Zhao X, Schalch J, Zhang J, Seren HR, Duan G, Averitt RD, et al. Electromechanically Tunable Metasurface Transmission Waveplate at Terahertz Frequencies. Optica (2018) 5(3):303-10. doi:10.1364/ optica.5.000303

29. Luo X, Hu F, and Li G. Dynamically Reversible and strong Circular Dichroism Based on Babinet-Invertible Chiral Metasurfaces. Opt Lett (2021) 46(6): 1309-12. doi:10.1364/ol.421016

30. Liu W, Hu B, Huang Z, Guan H, Li H, Wang X, et al. Graphene-enabled Electrically Controlled Terahertz Meta-Lens. Photon Res (2018) 6(7):703-8. doi:10.1364/prj.6.000703

31. Huang J, Guan H, Hu B, Wang G, Liu W, Wang Z, et al. Enhanced Terahertz Focusing for a Graphene-Enabled Active Metalens. Opt Express (2020) 28(23): 35179-91. doi:10.1364/oe.409746

32. Zhang Y, Feng Y, Zhu B, Zhao J, and Jiang T. Graphene Based Tunable Metamaterial Absorber and Polarization Modulation in Terahertz Frequency. Opt Express (2014) 22(19):22743-52. doi:10.1364/ oe.22.022743
Conflict of Interest: The authors declare that the research was conducted in the absence of any commercial or financial relationships that could be construed as a potential conflict of interest.

Publisher's Note: All claims expressed in this article are solely those of the authors and do not necessarily represent those of their affiliated organizations, or those of the publisher, the editors and the reviewers. Any product that may be evaluated in this article, or claim that may be made by its manufacturer, is not guaranteed or endorsed by the publisher.

Copyright (c) 2021 Wang, Hu, Khan and Zhang. This is an open-access article distributed under the terms of the Creative Commons Attribution License (CC BY). The use, distribution or reproduction in other forums is permitted, provided the original author(s) and the copyright owner(s) are credited and that the original publication in this journal is cited, in accordance with accepted academic practice. No use, distribution or reproduction is permitted which does not comply with these terms. 